\title{
Large-scale fluctuations and particle diffusion across external magnetic field in turbulent plasmas
}

\author{
A.Zagorodny, I.Holod \\ Bogolyubov Institute for Theoretical Physics, \\ 14b Metrolohichna Str., 03143 Kiev, Ukraine
}

Received April 17, 2000

\begin{abstract}
Kinetic theory of electromagnetic fluctuations in turbulent plasmas in the external magnetic field has been worked out with regard for the effect of fluid-like random motions on fluctuation dynamics. The dielectric response functions and correlation functions of the Langevin sources for the system under consideration are calculated and general relations for fluctuation spectra are derived. Fluctuations associated with the diffusive particle motion across the external magnetic field are studied in detail.
\end{abstract}

Key words: turbulent diffusion, Fokker-Planck equation, fluctuations

PACS: $05.40 .+j, 52.35 . R a, 25.65 . F f$

\section{Introduction}

Studies of density fluctuations associated with large-scale diffusive motion of plasma particles across the external magnetic field are of great importance in view of their application to non-contact diagnostics of fusion and geophysical plasmas. In spite of a considerable progress in such studies, many problems of the theory of electromagnetic fluctuations in plasmas still require their solution. First of all, this concerns the description of fluctuations in turbulent plasmas with regard for the effect of fluid-like random perturbations. The problem is that the traditional theory of electromagnetic fluctuations in stable stationary plasmas cannot be applied in this case. Since an appropriate generalization of the consistent theory of fluctuations within the microscopic description has not yet been done, various phenomenological approaches to this problem seem to be rather promising. In particular, one may assume that the statistical dependence of microscopic and large-scale motion is negligible and perform averaging over these motions independently, using appropriate structure factors. So, the next problem herein is to describe (or postulate) the statistical properties of turbulent perturbations. Making some assumptions regarding the physical nature of large-scale correlations (diffusive with a convective charac- 
ter of perturbations) it is possible to introduce the structure factor for turbulent pulsations. Such an approach was successfully applied to the interpretation of the scattering experiments with both fusion and geophysical plasmas [1,2]. Recently, a similar approach was used to describe the fluctuations associated with particle diffusion across the external magnetic field [3]. Within such phenomenological approach however, it is impossible to explicitly take into account the effects produced by the diffusion in the velocity space. At the same time these effects could be the primary reason for turbulent diffusion, since just the turbulent fields play the role of random forces producing additional stochastization.

The purpose of our paper is to describe electromagnetic fluctuations in turbulent plasmas in the external magnetic field treating the turbulent fields as a source of fluid-like motions in plasmas.

\section{Basic set of equations}

We study a plasma with stationary turbulence assuming that the characteristic time of turbulent field change $\tau_{\mathrm{T}}$ is much shorter than the correlation time for the fluctuations under consideration. Treating turbulent fields as a source of large scale perturbations it is possible to introduce these fields into the Langevin equations describing random particle (volume element) motion

$$
\begin{aligned}
\frac{\mathrm{d} \mathbf{r}}{\mathrm{d} t} & =\mathbf{v}, \\
\frac{\mathrm{d} v_{\mathrm{i}}}{\mathrm{d} t} & =-\beta_{\mathrm{i}} v_{\mathrm{i}}+\frac{1}{m}\left(F_{\mathrm{i}}^{\text {ext }}+\widetilde{F}_{\mathrm{i}}\right)+\frac{1}{m} \delta F_{i \mathrm{~T}},
\end{aligned}
$$

where $\beta_{\mathrm{i}}$ is the friction coefficient for a particle moving in the $i$-th direction, $\mathbf{F}^{\text {ext }}$ and $\widetilde{\mathbf{F}}$ are the forces associated with external and intrinsic plasma fields (the latter is smoothened over physically infinitesimal interval $\left.\tau_{\mathrm{ph}} \gg \tau_{\mathrm{T}}\right), \delta \mathbf{F}_{\mathrm{T}}$ is the Langevin force produced by the turbulent field. Statistical properties of the Langevin sources are given by

$$
\begin{aligned}
\left\langle\delta F_{i T}\right\rangle & =0 \\
\left\langle\delta F_{i T}(t) \delta F_{j T}\left(t^{\prime}\right)\right\rangle & =2 D_{i j} \delta\left(t-t^{\prime}\right) .
\end{aligned}
$$

Here, the angular brackets mean the statistical averaging which is equivalent to the time averaging over the interval $\tau \gtrsim \tau_{\mathrm{ph}} \gg \tau_{\mathrm{T}}$. Introducing equation (4) we assume that the Langevin forces are correlated within $\tau \lesssim \tau_{\text {ph }}$ only.

As was shown by Chandrasekhar [4] the Langevin equations (1)-(4) generate the generalized Liouville equation. Using this equation it is easy to show that in the case under consideration the equation for the microscopic phase density smoothened over $\tau_{\text {ph }}$ can be written as

$$
\left\{\frac{\partial}{\partial t}+\mathbf{v} \frac{\partial}{\partial \mathbf{r}}+\frac{1}{m}\left(\mathbf{F}^{\mathrm{ext}}+\widetilde{\mathbf{F}}\right) \frac{\partial}{\partial \mathbf{v}}\right\} \widetilde{N}(X, t)=\frac{\partial}{\partial v_{\mathrm{i}}}\left(\beta_{\mathrm{i}} v_{\mathrm{i}} \tilde{N}(X, t)+D_{i j} \frac{\partial \widetilde{N}(X, t)}{\partial v_{j}}\right) .
$$


Here, $\widetilde{N}(X, t)$ is the smoothened microscopic phase density

$$
\begin{aligned}
\widetilde{N}(X, t) & =\frac{1}{\tau_{\mathrm{ph}}} \int_{\tau-\frac{\tau_{\mathrm{ph}}}{2}}^{\tau+\frac{\tau_{\mathrm{ph}}}{2}} \mathrm{~d} t^{\prime} N(X, t), \\
N(X, t) & =\frac{1}{n} \sum_{\mathrm{i}} \delta\left(X-X_{\mathrm{i}}(t)\right),
\end{aligned}
$$

$X \equiv(\mathbf{r}, \mathbf{v}), X_{\mathrm{i}}(t) \equiv\left(r_{\mathrm{i}}(t), v_{\mathrm{i}}(t)\right)$ is the phase trajectory, the quantity $D_{i j}$ can be treated as a diffusion coefficient in the velocity space.

Notice, that equation (5) can be also introduced by averaging of the equation for the microscopic phase density over $\tau_{\mathrm{ph}} \gg \tau_{\mathrm{T}}$ and using the Fokker-Planck representation for the collision term with the kinetic coefficient determined by the turbulent field correlation function [5]. In the general case kinetic coefficient $\beta_{\mathrm{i}}$ and $D_{i j}$ should be determined selfconsistently with regard for their temporary evolution [6]. In what follows the kinetic coefficients are assumed to be known. In the case under consideration we also suggest that the turbulence is isotropic in the directions perpendicular to the external magnetic field and that the velocity dependence of the kinetic coefficients may be neglected. Within such a model, equation (5) has the form

$$
\widehat{L}_{0} \widetilde{N}(X, t)+\frac{e}{m} \widetilde{\mathbf{E}} \frac{\partial \widetilde{N}(X, t)}{\partial \mathbf{v}}=0,
$$

here

$$
\begin{aligned}
\widehat{L}_{0}= & \frac{\partial}{\partial t}+\mathbf{v} \frac{\partial}{\partial \mathbf{r}}+[\mathbf{v} \times \Omega] \frac{\partial}{\partial \mathbf{v}}-\frac{\partial}{\partial v_{\|}}\left(\beta_{\|} v_{\|}+D_{\|} \frac{\partial}{\partial v_{\|}}\right) \\
& -\frac{\partial}{\partial \mathbf{v}_{\perp}}\left(\beta_{\perp} \mathbf{v}_{\perp}+D_{\perp} \frac{\partial}{\partial \mathbf{v}_{\perp}}\right), \\
& \boldsymbol{\Omega}=(0,0, \Omega), \quad \Omega=\frac{e B_{0}}{m c} ; \quad \mathbf{v} \equiv\left(\mathbf{v}_{\perp}, v_{\|}\right),
\end{aligned}
$$

$\beta_{\|}, \beta_{\perp}, D_{\|}$and $D_{\perp}$ are the relevant kinetic coefficients.

Averaging equation (7) over the Liouville distribution one has

where

$$
\widehat{L}_{0} f(X, t)+\frac{e}{m}\langle\mathbf{E}\rangle \frac{\partial f(X, t)}{\partial \mathbf{v}}=-\frac{e}{m} \frac{\partial}{\partial \mathbf{v}}\langle\delta \widetilde{\mathbf{E}}(\mathbf{r}, t) \delta \widetilde{N}(X, t)\rangle,
$$

$$
\begin{gathered}
f(X, t) \equiv\langle\widetilde{N}(X, t)\rangle, \quad \delta \widetilde{N}(X, t)=\widetilde{N}(X, t)-f(X, t) ; \\
\delta \mathbf{E}(\mathbf{r}, t)=\widetilde{\mathbf{E}}(\mathbf{r}, t)-\langle\mathbf{E}\rangle .
\end{gathered}
$$

Equations (7), (9) generate the following linearized equations

$$
\begin{gathered}
\widehat{L}_{0} \delta \widetilde{N}(X, t)+\frac{e}{m} \delta \mathbf{E}(\mathbf{r}, t) \frac{f(X, t)}{\partial \mathbf{v}}=0, \\
\operatorname{div} \delta \widetilde{\mathbf{E}}(\mathbf{r}, t)=4 \pi e n \int \mathrm{d} \mathbf{v} \delta \widetilde{N}(X, t) .
\end{gathered}
$$

These equations describe fluctuation dynamics with regard for the influence of turbulent fields producing diffusion in the velocity space. 


\section{Transition probability approach to the theory of large-scale fluctuations}

The formal solution of equation (10) consists of two parts

$$
\delta \widetilde{N}(X, t)=\delta \widetilde{N}^{(0)}(X, t)-\frac{e}{m} \int_{-\infty}^{t} \mathrm{~d} t^{\prime} \int \mathrm{d} X^{\prime} W\left(X, X^{\prime} ; t-t^{\prime}\right) \delta \widetilde{\mathbf{E}}\left(\mathbf{r}^{\prime}, t^{\prime}\right) \frac{\partial f\left(v^{\prime}\right)}{\partial \mathbf{v}^{\prime}}
$$

where $\delta \widetilde{N}^{(0)}(X, t)$ is the fluctuation in the relevant system with no selfconsistent interaction (this part is associated with general solution of the homogeneous equation), i.e.

$$
\widehat{L}_{0} \delta \widetilde{N}^{(0)}(X, t)=0
$$

and the second part is the partial solution of the inhomogeneous equation written in terms of particle transition probability satisfying the equation

$$
\widehat{L}_{0} W\left(X, X^{\prime} ; t-t^{\prime}\right)=0
$$

with the initial condition

$$
W\left(X, X^{\prime}, 0\right)=\delta\left(X-X^{\prime}\right) .
$$

Substituting the solution (12) into equation (11) we obtain

$$
\begin{aligned}
& \operatorname{div} \delta \widetilde{\mathbf{E}}(\mathbf{r}, t)+\frac{4 \pi e^{2} n}{m} \int_{-\infty}^{t} \mathrm{~d} t^{\prime} \int \mathrm{d} X^{\prime} \mathrm{d} \mathbf{v}^{\prime} W\left(X, X^{\prime}, t-t^{\prime}\right) \delta \widetilde{\mathbf{E}}\left(\mathbf{r}^{\prime}, t^{\prime}\right) \frac{\partial f\left(v^{\prime}\right)}{\partial \mathbf{v}^{\prime}}= \\
& \quad=4 \pi e n \int \mathrm{d} \mathbf{v} \delta \widetilde{N}^{(0)}(X, t) .
\end{aligned}
$$

Thus we see that the quantity $\delta \widetilde{N}^{(0)}(X, t)$ can be treated as the Langevin sources of fluctuation field $\delta \widetilde{\mathbf{E}}(\mathbf{r}, t)$. Correlation function for such sources can be calculated using (13)-(15). In particular, as follows from these equations

$$
\delta N^{(0)}(X, t)=\int \mathrm{d} X^{\prime \prime} W\left(X, X^{\prime \prime}, t-t^{\prime}\right) \delta N^{(0)}\left(X^{\prime \prime}, t^{\prime}\right), \quad t>t^{\prime},
$$

that in turn gives

$$
\left\langle\delta N^{(0)}(X, t) \delta N^{(0)}\left(X^{\prime}, t^{\prime}\right)\right\rangle=\int \mathrm{d} X^{\prime \prime} W\left(X, X^{\prime \prime}, t-t^{\prime}\right)\left\langle\delta N^{(0)}\left(X^{\prime \prime}, t^{\prime}\right) \delta N^{(0)}\left(X^{\prime}, t^{\prime}\right)\right\rangle
$$

with regard for the fact that

$$
\left\langle\delta \widetilde{N}^{(0)}(X, t) \delta \widetilde{N}^{(0)}\left(X^{\prime}, t\right)\right\rangle=\frac{1}{n} f(X, t) \delta\left(X-X^{\prime}\right)+\widetilde{g}\left(X, X^{\prime}, t\right),
$$


where $\widetilde{g}\left(X, X^{\prime}, t\right)$ is the static binary correlation function of physically infinitesimal volume element,

$$
\begin{aligned}
\left\langle\delta \widetilde{N}^{(0)}(X, t) \delta \widetilde{N}^{(0)}\left(X^{\prime}, t^{\prime}\right)\right\rangle=\frac{1}{n}\{ & f\left(X^{\prime}, t^{\prime}\right) W\left(X, X^{\prime}, t-t^{\prime}\right) \Theta\left(t-t^{\prime}\right) \\
& \left.+f(X, t) W\left(X^{\prime}, X, t^{\prime}-t\right) \Theta\left(t^{\prime}-t\right)\right\} \\
+ & \int \mathrm{d} X^{\prime \prime}\left\{W\left(X, X^{\prime \prime}, t-t^{\prime}\right) \widetilde{g}\left(X^{\prime \prime}, X^{\prime}, t^{\prime}\right) \Theta\left(t-t^{\prime}\right)\right. \\
+ & \left.W\left(X^{\prime}, X^{\prime \prime}, t^{\prime}-t\right) \widetilde{g}\left(X, X^{\prime \prime}, t\right) \Theta\left(t^{\prime}-t\right)\right\}
\end{aligned}
$$

If $\widetilde{g}\left(X, X^{\prime}, t\right)$ could be neglected

$$
\begin{aligned}
\left\langle\delta \widetilde{N}^{(0)}(X, t) \delta \widetilde{N}^{(0)}\left(X^{\prime}, t^{\prime}\right)\right\rangle=\frac{1}{n}\{ & f\left(X^{\prime}, t\right) W\left(X, X^{\prime}, t-t^{\prime}\right) \Theta\left(t-t^{\prime}\right) \\
& \left.+f(X, t) W\left(X^{\prime}, X, t^{\prime}-t\right) \Theta\left(t^{\prime}-t\right)\right\} .
\end{aligned}
$$

In the case of spatially homogeneous stationary turbulence in the $\mathbf{k} ; \omega$-representation equations (16), (21) have the form

$$
\begin{aligned}
\mathrm{i} \mathbf{k} \varepsilon(\mathbf{k}, \omega) \delta \mathbf{E}_{\mathbf{k} \omega} & =4 \pi \delta \widetilde{\rho}_{\mathbf{k} \omega}^{(0)} \\
\left\langle\delta N^{(0)}(\mathbf{v}), \delta N^{(0)}\left(\mathbf{v}^{\prime}\right)\right\rangle_{\mathbf{k} \omega} & =\frac{1}{n} f\left(\mathbf{v}^{\prime}\right) W_{\mathbf{k} \omega}\left(\mathbf{v}, \mathbf{v}^{\prime}\right)+f(\mathbf{v}) W_{\mathbf{k} \omega}\left(\mathbf{v}^{\prime}, \mathbf{v}\right)
\end{aligned}
$$

where

$$
\begin{aligned}
\varepsilon(\mathbf{k}, \omega) & =1+\sum \chi(\mathbf{k}, \omega), \\
\chi(\mathbf{k}, \omega) & =-\mathrm{i} \frac{4 \pi e n}{k^{2}} \int \mathrm{d} \mathbf{v} \int \mathrm{d}^{\prime} W_{\mathbf{k} \omega}\left(\mathbf{v}, \mathbf{v}^{\prime}\right) \mathbf{k} \frac{\partial f}{\partial \mathbf{v}}, \\
W_{\mathbf{k}, \omega}\left(\mathbf{v}, \mathbf{v}^{\prime}\right) & =\int_{0}^{\infty} \mathrm{d} \tau \int \mathrm{d} \mathbf{R} \mathrm{e}^{-\mathrm{i} \mathbf{k} \mathbf{R}+\mathrm{i} \omega \tau} W\left(X, X^{\prime}, \tau\right) .
\end{aligned}
$$

Equations (22)-(24) give the solution of the problem under consideration. Using these equations it is possible to calculate correlation functions of any electromagnetic quantities. For example,

$$
\left\langle\delta n_{\mathrm{e}}^{2}\right\rangle_{\mathbf{k} \omega}=\left|\frac{1+\chi_{\mathrm{i}}(\mathbf{k}, \omega)}{\varepsilon(\mathbf{k}, \omega)}\right|^{2}\left\langle\delta n_{\mathrm{e}}^{2}\right\rangle_{\mathbf{k} \omega}^{(0)}+Z_{\mathrm{i}}\left|\frac{\chi_{\mathrm{e}}(\mathbf{k}, \omega)}{\varepsilon(\mathbf{k}, \omega)}\right|^{2}\left\langle\delta n_{\mathrm{i}}^{2}\right\rangle_{\mathbf{k} \omega}^{(0)}
$$

where

$$
\left\langle\delta n^{2}\right\rangle_{\mathbf{k} \omega}^{(0)}=e^{2} n \int \mathrm{d} \mathbf{v} \int \mathrm{d} \mathbf{v}^{\prime} W_{\mathbf{k} \omega}\left(\mathbf{v}, \mathbf{v}^{\prime}\right) f\left(\mathbf{v}^{\prime}\right)+\text { c.c. } .
$$

Equation (25) has the same form as that for fluctuation spectra in a stable plasma (see, for example, [7]), however, the quantities $\varepsilon(\mathbf{k}, \omega)$ and $\left\langle\delta n^{2}\right\rangle_{\mathbf{k} \omega}^{(0)}$ are determined by the renormalized transition probability $W\left(X, X^{\prime}, \tau\right)$ calculated based on the equation (7) with regard for the action of turbulent fields on particle dynamics. Thus, the next problem in our calculations is to find this quantity in explicit form. 


\section{Renormalized transition probability for a plasma in an exter- nal magnetic field}

Taking into account the spatial symmetry properties of the operator $\widehat{L}_{0}$ (in particular, independency of the motions along and across the external magnetic field) the solution of equation (14) with the initial condition (15) can be represented in the following form

$$
W\left(X, X^{\prime}, \tau\right)=W_{\perp}\left(X_{\perp}, X_{\perp}^{\prime}, \tau\right) W_{\|}\left(X_{\|}, X_{\|}^{\prime}, \tau\right)
$$

where $W_{\perp}\left(X_{\perp}, X_{\perp}^{\prime}, \tau\right)$ and $W_{\|}\left(X_{\|}, X_{\|}, \tau\right)$ are the probabilities of particle transition across and along the external magnetic field, respectively, $X_{\perp} \equiv\left(\mathbf{r}_{\perp}, \mathbf{v}_{\perp}\right), X_{\|} \equiv$ $\left(z, v_{\|}\right)$. As it is easy to show, these transition probabilities satisfy the equations

$$
\begin{gathered}
\left\{\frac{\partial}{\partial t}+v_{\|} \frac{\partial}{\partial z}-\frac{\partial}{\partial v_{\|}}\left(\beta_{\|} v_{\|}+D_{\|} \frac{\partial}{\partial v_{\|}}\right)\right\} W_{\|}\left(X_{\|}, X_{\|}^{\prime} ; \tau\right)=0 \\
\left\{\frac{\partial}{\partial t}+v_{\perp} \frac{\partial}{\partial \mathbf{r}_{\perp}}+\left[\mathbf{v}_{\perp} \times \Omega\right] \frac{\partial}{\partial \mathbf{v}_{\perp}}-\frac{\partial}{\partial \mathbf{v}_{\perp}}\left(\beta_{\perp} \mathbf{v}_{\perp}+D_{\perp} \frac{\partial}{\partial \mathbf{v}_{\perp}}\right)\right\} W_{\perp}\left(X_{\perp}, X_{\perp}^{\prime} ; \tau\right)=0
\end{gathered}
$$

with the initial conditions of the type (15).

Solution of equation (28) was done by Chandrasekhar in the well-known paper [4]. This solution is as follows

$$
W_{\|}\left(X_{\|}, X_{\|}^{\prime}, \tau\right)=\frac{\mathrm{e}^{\beta_{\|} \tau}}{2 \pi \triangle_{\|}} \exp \left\{-\frac{1}{2 \triangle_{\|}}\left[a_{\|} \rho_{\|}^{2}+b_{\|} P_{\|}^{2}+2 h_{\|} \rho_{\|} P_{\|}\right]\right\},
$$

where

$$
\begin{gathered}
\rho_{\|}=\mathrm{e}^{-\beta_{\|} \tau} v_{\|}-v_{\|}^{\prime}, \quad P_{\|}=z-z^{\prime}+\frac{v_{\|}-v_{\|}^{\prime}}{\beta_{\|}}, \\
a_{\|}=\frac{2 D_{\|}}{\beta_{\|}^{2}} \tau, \quad b_{\|}=\frac{2 D_{\|}}{\beta_{\|}^{2}}\left(\mathrm{e}^{2 \beta_{\|} \tau}-1\right), \quad h_{\|}=\frac{2 D_{\|}}{\beta_{\|}^{2}}\left(1-\mathrm{e}^{\beta_{\|} \tau}\right) .
\end{gathered}
$$

As regards equation (29), the way of obtaining its solution is given below. Let us start from the integration of the equation of motion for charged particle moving in viscous media across the external magnetic field

$$
\ddot{x}=\Omega \dot{y}-\beta_{\perp} \dot{x}=\dot{v}_{x}, \quad \ddot{y}=-\Omega \dot{x}-\beta_{\perp} \dot{y}=\dot{v}_{y} .
$$

These equations can be easily integrated. The integrals of motion of equations (32) are

$$
\begin{aligned}
& \rho_{x}=\mathrm{e}^{\beta_{\perp} \tau}\left(v_{x} \cos \Omega \tau-v_{y} \sin \Omega \tau\right)-v_{x}^{\prime}, \\
& \rho_{x}=\mathrm{e}^{\beta_{\perp} \tau}\left(v_{x} \sin \Omega \tau+v_{y} \cos \Omega \tau\right)-v_{y}^{\prime}, \\
& P_{x}=x-x^{\prime}+\frac{\beta_{\perp}\left(v_{x}-v_{x}^{\prime}\right)+\Omega\left(v_{y}-v_{y}^{\prime}\right)}{\Omega^{2}+\beta_{\perp}^{2}}, \\
& P_{x}=y-y^{\prime}+\frac{\beta_{\perp}\left(v_{y}-v_{y}^{\prime}\right)-\Omega\left(v_{x}-v_{x}^{\prime}\right)}{\Omega^{2}+\beta_{\perp}^{2}} .
\end{aligned}
$$


In terms of the variables $\boldsymbol{\rho}_{\perp}$ and $\mathbf{P}_{\perp}$ the equation for the function

$$
w\left(X_{\perp}, X_{\perp}^{\prime}, \tau\right)=\mathrm{e}^{-2 \beta_{\perp} \tau} W_{\perp}\left(X_{\perp}, X_{\perp}^{\prime}, \tau\right),
$$

has the form

$$
\begin{aligned}
\frac{1}{D_{\perp}} \frac{\partial w}{\partial t}= & \mathrm{e}^{\beta \tau} \frac{\partial^{2} w}{\partial \boldsymbol{\rho}_{\perp}^{2}}+\frac{\partial^{2} w}{\partial \mathbf{P}_{\perp}^{2}}+\frac{2 \mathrm{e}^{\beta_{\perp} \tau}}{\Omega^{2}+\beta_{\perp}^{2}}\left(\beta_{\perp} \cos \Omega \tau-\Omega \sin \Omega \tau\right)\left(\frac{\partial^{2}}{\partial \rho_{x} \partial P_{x}}+\frac{\partial^{2}}{\partial \rho_{y} \partial P_{y}}\right) w \\
& +\frac{2 \mathrm{e}^{\beta_{\perp} \tau}}{\Omega^{2}+\beta_{\perp}^{2}}\left(\beta_{\perp} \sin \Omega \tau+\Omega \cos \Omega \tau\right)\left(\frac{\partial^{2}}{\partial \rho_{x} \partial P_{y}}-\frac{\partial^{2}}{\partial \rho_{y} \partial P_{x}}\right) w
\end{aligned}
$$

with the initial condition

$$
w\left(\boldsymbol{\rho}_{\perp}, \mathbf{P}_{\perp}, 0\right)=\delta\left(\boldsymbol{\rho}_{\perp}\right) \delta\left(\mathbf{P}_{\perp}\right) .
$$

Now let us recollect the lemma 11 from the Chandrasekhar's paper [4]. According to this lemma the solution of the equation

$$
\frac{\partial F}{\partial t}=\varphi^{2}(t) \frac{\partial^{2} F}{\partial x^{2}}+2 \varphi(t) \psi(t) \frac{\partial^{2} F}{\partial x \partial y}+\psi^{2}(t) \frac{\partial^{2} F}{\partial y^{2}}
$$

with the initial condition

$$
F(x, y, 0)=\delta(x) \delta(y)
$$

and arbitrary $\varphi(t)$ and $\psi(t)$ is given by

$$
F(x, y)=\frac{1}{2 \pi \triangle^{1 / 2}} \exp \left\{-\frac{1}{2 \triangle}\left(a x^{2}+2 b x y+b y^{2}\right)\right\}
$$

where

$$
\triangle=a b-h^{2}, \quad a=\int_{0}^{t} \mathrm{~d} t \psi^{2}(t), \quad h=-2 \int_{0}^{t} \mathrm{~d} t \varphi(t) \psi(t), \quad b=2 \int_{0}^{t} \mathrm{~d} t \psi^{2}(t) .
$$

As is seen, the power of exponent in equation (38) reproduced the structure of the right-hand part of equation (36). Using the analogy we can write the solution of equation (34) in the form

$$
w\left(\boldsymbol{\rho}_{\perp}, \mathbf{P}_{\perp}, \tau\right)=\frac{1}{4 \pi^{2} \triangle_{\perp}} \exp \left\{-\frac{1}{2 \triangle_{\perp}}\left[a_{\perp} \boldsymbol{\rho}_{\perp}^{2}+b_{\perp} \mathbf{P}_{\perp}^{2}+2 h_{\perp} \boldsymbol{\rho}_{\perp} \mathbf{P}_{\perp}-2 q_{\perp} \mathbf{e}_{z}\left[\boldsymbol{\rho}_{\perp} \mathbf{P}_{\perp}\right]\right]\right\}
$$

where

$$
\begin{gathered}
\triangle_{\perp}=a_{\perp} b_{\perp}-h_{\perp}^{2}-q_{\perp}^{2}, \quad a_{\perp}=\frac{2 D_{\perp} \tau}{\Omega_{\perp}^{2}+\beta_{\perp}^{2}}, \quad b_{\perp}=\frac{2 D_{\perp}}{\beta_{\perp}}\left(\mathrm{e}^{2 \beta_{\perp} \tau}-1\right), \\
h_{\perp}=\frac{2 D_{\perp}}{\Omega_{\perp}^{2}+\beta_{\perp}^{2}}\left(1-\mathrm{e}^{\beta_{\perp} \tau} \cos \Omega \tau\right), \quad q_{\perp}=-\frac{2 D_{\perp}}{\Omega_{\perp}^{2}+\beta_{\perp}^{2}} \mathrm{e}^{\beta_{\perp} \tau} \sin \Omega \tau .
\end{gathered}
$$


Thus the solution of equation (29) can be written as

$$
\begin{aligned}
& W_{\perp}\left(X_{\perp}, X_{\perp}^{\prime}, \tau\right)= \\
& \quad=\frac{\mathrm{e}^{2 \beta_{\perp} \tau}}{4 \pi^{2} \triangle_{\perp}} \exp \left\{-\frac{1}{2 \triangle_{\perp}}\left[a_{\perp} \boldsymbol{\rho}_{\perp}^{2}+b_{\perp} \mathbf{P}_{\perp}^{2}+2 h_{\perp} \boldsymbol{\rho}_{\perp} \mathbf{P}_{\perp}-2 q_{\perp} \mathbf{e}_{z}\left[\boldsymbol{\rho}_{\perp} \mathbf{P}_{\perp}\right]\right]\right\} .
\end{aligned}
$$

Together with equation (30) this equation gives the generalization of the Chandrasekhar's solution to the case of a charged particle moving in an external magnetic field.

Using equation (42) it is possible to calculate a mean and a mean-square particle displacements across the external magnetic field

$$
\begin{aligned}
\left\langle\triangle \mathbf{r}_{\perp}^{2}\right\rangle_{\tau}= & \int \mathrm{d} \triangle \mathbf{r}_{\perp} \int \mathrm{d} \triangle \mathbf{v}_{\perp} \int \mathrm{d} \mathbf{v}_{\perp} \triangle \mathbf{r}_{\perp}^{2} W_{\perp}\left(\mathbf{r}_{\perp}+\triangle \mathbf{r}_{\perp}, \mathbf{v}_{\perp}+\triangle \mathbf{r}_{\perp}, \mathbf{r}_{\perp}, \mathbf{v}_{\perp}, \tau\right) f\left(v_{\perp}\right) \\
= & \frac{D_{\perp}}{\Omega^{2}+\beta^{2}}\left\{\tau+\frac{1}{2 \beta_{\perp}}\left(1-\mathrm{e}^{-2 \beta_{\perp} \tau}\right)\right. \\
& \left.\quad-\frac{2}{\Omega^{2}+\beta^{2}}\left[\beta_{\perp}-\mathrm{e}^{-\beta_{\perp} \tau}\left(\beta_{\perp} \cos \Omega \tau-\Omega \sin \Omega \tau\right)\right]\right\} \\
& +\frac{\left\langle v_{\perp}^{2}\right\rangle}{\Omega^{2}+\beta^{2}}\left[\left(1-\mathrm{e}^{-\beta_{\perp} \tau} \cos \Omega \tau\right)^{2}+\mathrm{e}^{-2 \beta_{\perp} \tau} \sin \Omega \tau\right] \\
\left\langle v_{\perp}^{2}\right\rangle= & \int d \mathbf{v}_{\perp} v_{\perp}^{2} f\left(v_{\perp}\right) .
\end{aligned}
$$

In the case of the Maxwellian velocity distribution

$$
\left\langle v_{\perp}^{2}\right\rangle=2(T / m)=2 S_{\perp}^{2}, \quad D_{\perp}=S_{\perp}^{2} \beta_{\perp}
$$

and equation (43) reduced to the result obtained in [3]

$$
\left\langle\triangle \vec{r}_{\perp}^{2}\right\rangle_{\tau}=\frac{S_{\perp}^{2}}{\Omega^{2}+\beta^{2}}\left\{\beta_{\perp} \tau+\frac{\Omega^{2}-\beta^{2}}{\Omega^{2}+\beta^{2}}\left(1-\mathrm{e}^{-\beta_{\perp} \tau} \cos \Omega \tau\right)-\frac{2 \beta_{\perp} \Omega}{\Omega^{2}+\beta^{2}} \mathrm{e}^{-\beta_{\perp} \tau} \sin \Omega \tau\right\} .
$$

\section{Dielectric response function and correlation functions of the Langevin sources for a plasma in an external magnetic field}

Using general relations (24), (26) and an explicit expression for the transition probability (equations $(27),(30),(42))$ it is possible to calculate the response functions and correlation functions of the Langevin sources for a plasma in an external magnetic field with regard for large-scale diffusive motions.

What is important is that we have no restrictions regarding the values of kinetic coefficients and characteristic fluctuation times. This mean that we can apply the obtained relations for the description of fluctuations and electromagnetic properties of plasmas in various regimes, in particular to study the effects of diffusion in velocity and real spaces. 
For example, in the general case

$$
\begin{aligned}
\chi(k, \omega)= & -\mathrm{i} \frac{\omega_{p}^{2}}{k^{2}} \int_{0}^{\infty} \mathrm{d} \tau \int \mathrm{d} \mathbf{v} \sum_{m} J_{m}(\xi)\left\{\left[\frac{m \widetilde{\Omega}}{v_{\perp}} \cos \psi J_{m}(\xi)\right.\right. \\
& \left.\left.+\mathrm{i} k_{\perp} \sin \psi J_{m}^{\prime}(\xi)\right] \frac{\partial f}{\partial v_{\perp}}+J_{m}(\xi) k_{\|} \frac{\partial f}{\partial v_{\|}}\right\} \\
& \times \exp \left\{-\left(\frac{k_{\perp}^{2} D_{\perp}}{\widetilde{\Omega}^{2}} \Phi_{\perp}(\tau)+\frac{k_{\|}^{2} D_{\|}}{\widetilde{\beta}_{\|}{ }^{2}} \Phi_{\|}(\tau)\right)\right. \\
& \left.+\mathrm{i}\left[(\omega-m \Omega) \tau-\frac{k_{\|} v_{\|}}{\beta_{\|}}\left(1-\mathrm{e}^{-\beta_{\|} \tau}\right)\right]\right\} \\
\left\langle\delta n^{2}\right\rangle_{\mathbf{k} \omega}^{(0)}= & n \int_{0}^{\infty} \mathrm{d} \tau \int \mathrm{d} \mathbf{v} \sum_{m} J_{m}(\xi) J_{m}(\widetilde{\xi}) f(\mathbf{v}) \\
& \times \exp \left\{-\left(\frac{k_{\perp}^{2}}{\widetilde{\omega}^{2}} \Phi_{\perp}(\tau)+\frac{k_{\|}^{2}}{\widetilde{\beta}_{\|}^{2}} \Phi_{\|}(\tau)\right)\right. \\
& \left.+\mathrm{i}\left[(\omega-m \Omega) \tau-\frac{k_{\|} v_{\|}}{\beta_{\|}}\left(1-\mathrm{e}^{-\beta_{\|} \tau}\right)\right]\right\}+ \text { c.c. },
\end{aligned}
$$

where

$$
\begin{aligned}
& \Phi_{\perp}(\tau)=\tau+\frac{1}{2 \beta \perp}\left(1-\mathrm{e}^{-2 \beta_{\perp} \tau}\right)-\frac{2}{\widetilde{\Omega}^{2}}\left[\beta_{\perp}-\mathrm{e}^{-\beta_{\perp} \tau}\left(\beta_{\perp} \cos \Omega \tau-\Omega \sin \Omega \tau\right)\right] \\
& \Phi_{\|}(\tau)=\tau+\frac{1}{2 \beta_{\|}}\left(1-\mathrm{e}^{-2 \beta_{\|} \tau}\right)-\frac{2}{\beta_{\|}}\left[1-\mathrm{e}^{-\beta_{\|} \tau}\right] \\
& \widetilde{\Omega}^{2}=\Omega^{2}+\beta_{\perp}^{2}, \quad \cos \psi=\frac{\Omega}{\sqrt{\Omega^{2}+\beta_{\perp}^{2}}}, \quad \sin \psi=\frac{\beta_{\perp}}{\sqrt{\Omega^{2}+\beta_{\perp}^{2}}} \\
& \xi=\frac{k_{\perp} v_{\perp}}{\widetilde{\Omega}}, \quad \widetilde{\xi}=\frac{k_{\perp} v_{\perp}}{\widetilde{\Omega}} \mathrm{e}^{-\beta_{\perp} \tau} .
\end{aligned}
$$

Equations (46)-(48) make it possible to recover various particular cases. For instance, for $D_{\|}=D_{\perp}=0, \beta_{\|}=\beta_{\perp}=0$ one has the well-known results for the Vlasov plasma. For $\beta_{\perp} \sim \beta_{\|} \sim 0$ (minor friction) and $D_{\|}=0$ (no diffusion along the magnetic field)

$$
\chi(\mathbf{k}, \omega)=\frac{\omega_{p}^{2}}{k^{2}} \sum_{n} \sum_{m}(\mathrm{i})^{m} \int \mathrm{d} \mathbf{v} J_{m}^{2}(\xi) I_{n}\left(\frac{\widetilde{\nu}_{0}}{\Omega}\right) \frac{\frac{n \Omega}{v_{\perp}} \frac{\partial f}{\partial v_{\perp}}+k_{\|} \frac{\partial f}{\partial v_{\|}}}{\omega-k_{\|} v_{\|}-(n+m) \Omega+\mathrm{i} \widetilde{\nu}_{0}},
$$

where

$$
\widetilde{\nu}_{0}=\frac{k_{\perp}^{2} D_{\perp}}{\Omega^{2}}
$$


In the case $\beta_{\|}=0, D_{\|}=0$ and $\beta_{\perp} \gg \omega$ equations (46), (47) give

$$
\begin{aligned}
\chi(\mathbf{k}, \omega) & =\frac{\omega_{p}^{2}}{k^{2}} \int \mathrm{d} \mathbf{v} \frac{J_{0}(\xi) k_{\|} \frac{\partial f}{\partial v_{\|}}+\mathrm{i} \frac{k_{\perp} \beta_{\perp}}{\Omega} J_{0}^{\prime}(\xi) \frac{\partial f}{\partial v_{\perp}}}{\omega-k_{\|} v_{\|}+\mathrm{i} \widetilde{\nu}} \\
\left\langle\delta n^{2}\right\rangle_{\mathbf{k}, \omega}^{(0)} & =n \int \mathrm{d} \mathbf{v} \frac{J_{0}(\xi) f(v)}{\omega-k_{\|} v_{\|}+\mathrm{i} \widetilde{\nu}}+\text { c.c., } \quad \widetilde{\nu}=\frac{k_{\perp}^{2} D_{\perp}}{\widetilde{\Omega}^{2}} .
\end{aligned}
$$

In many cases of scattering experiments it is necessary to consider the limit $k_{\|}=0$. Assuming that the velocity distribution is the Maxwellian distribution, it is possible to show that in the above limit

$$
\begin{aligned}
\chi(\mathbf{k}, \omega)= & \mathrm{i} \frac{\omega_{p}^{2}}{k^{2} S_{\perp}^{2}} \int_{0}^{\infty} \mathrm{d} \tau \sum_{m} \mathrm{e}^{-\frac{\widetilde{\beta}}{2}\left(1+\mathrm{e}^{-2 \beta_{\perp} \tau}\right)}\left\{m \Omega I_{m}\left(\widetilde{\beta} \mathrm{e}^{-\beta_{\perp} \tau}\right)\right. \\
& \left.+\mathrm{i} \widetilde{\beta} \beta_{\perp}\left[I_{m}^{\prime}\left(\widetilde{\beta} \mathrm{e}^{-\beta_{\perp} \tau}\right) \mathrm{e}^{-\beta_{\perp} \tau}-I_{m}\left(\widetilde{\beta} \mathrm{e}^{-\beta_{\perp} \tau}\right)\right]\right\} \\
& \times \exp \left\{-\frac{k_{\perp}^{2} D_{\perp}}{\widetilde{\Omega}^{2}} \Phi_{\perp}(\tau)+\mathrm{i}(\omega-m \Omega) \tau\right\} \\
\left\langle\delta n^{2}\right\rangle_{\mathbf{k} \omega}^{(0)}= & n \int_{0}^{\infty} \mathrm{d} \tau \sum_{m} I_{m}\left(\widetilde{\beta} \mathrm{e}^{-\beta_{\perp} \tau}\right) \mathrm{e}^{-\frac{\widetilde{\beta}}{2}\left(1+\mathrm{e}^{-\beta_{\perp} \tau}\right)} \\
& \times \exp \left\{-\frac{k_{\perp}^{2} D_{\perp}}{\widetilde{\Omega}^{2}} \Phi_{\perp}(\tau)+\mathrm{i}(\omega-m \Omega) \tau\right\}
\end{aligned}
$$

where

$$
\widetilde{\beta}=\frac{k_{\perp}^{2} S_{\perp}^{2}}{\widetilde{\Omega}^{2}}
$$

In the diffusive regime $\left(\beta_{\perp} \gg \omega\right)$ these equations give

$$
\begin{aligned}
\chi(\mathbf{k}, \omega) & =\mathrm{i} \frac{\omega_{p}^{2} \beta_{\perp}}{\widetilde{\Omega}^{2}} \frac{\mathrm{e}^{-\frac{\widetilde{\beta}}{2}}}{\omega+\mathrm{i} k_{\perp}^{2} D_{r}}=\mathrm{i} \frac{k_{\mathrm{D}}^{2} D_{r}}{\omega+\mathrm{i} k_{\perp}^{2} D_{r}}, \\
\left\langle\delta n^{2}\right\rangle_{\mathbf{k} \omega}^{(0)} & =n \frac{2 k^{2} D_{r}}{\left|\omega+\mathrm{i} k_{\perp}^{2} D_{r}\right|^{2}} \mathrm{e}^{-\frac{\widetilde{\beta}}{2}} .
\end{aligned}
$$

Here,

$$
D_{r}=\frac{D_{\perp} \beta_{\perp}}{\widetilde{\Omega}^{2}}, \quad k_{\mathrm{D}}^{2}=\frac{4 \pi \mathrm{e}^{2} n}{T} \mathrm{e}^{-\frac{\widetilde{\beta}}{2}} .
$$

\section{Fluctuations associated with particle diffusion across an ex- ternal magnetic field in the system of interacting particles}

According to equation (14) the transition probability $W\left(X, X^{\prime}, \tau\right)$ describes particle motion (in particular, particle diffusion at $\beta \tau \gg 1$ ) disregarding the interaction 
between charged particles through a selfconsistent electric field. This means that the correlation functions of the type (23), (26) as well as the mean-square particle displacement can be used for describing the fluctuations and wave scattering in the cases of negligible effect of particle interaction only. In order to take into account collective effects it is necessary to use a general relation for the correlation function (25).

Let us consider some consequences of the combined interaction in the diffusion limit. In this case we can use equations (54) that leads to

$$
\begin{aligned}
\varepsilon(\mathbf{k}, \omega) & \simeq \frac{\mathrm{i}\left(k_{\mathrm{e}}^{2} D_{\mathrm{e}}+k_{\mathrm{i}}^{2} D_{\mathrm{i}}\right)}{\left(\omega+\mathrm{i} k^{2} D_{\mathrm{e}}\right)\left(\omega+\mathrm{i} k^{2} D_{\mathrm{i}}\right)}\left(\omega+\mathrm{i} k^{2} D_{\mathrm{A}}\right), \\
\chi_{\alpha}(\mathbf{k}, \omega) & =\frac{\mathrm{i} k_{\alpha}^{2} D_{\alpha}}{\omega+\mathrm{i} k^{2} D_{\alpha}}, \\
\left\langle\delta n_{\alpha}^{2}\right\rangle_{\mathbf{k} \omega}^{(0)} & =2 n_{\alpha} \frac{k^{2} D_{\alpha}}{\left|\omega+\mathrm{i} k^{2} D_{\alpha}\right|^{2}} .
\end{aligned}
$$

Here, we introduce the notation

$$
k_{\alpha}^{2} \equiv k_{D \alpha}^{2}=\frac{4 \pi e_{\alpha}^{2} n_{\alpha}}{T_{\alpha}}, \quad D_{\alpha} \equiv D_{r \alpha}=\frac{D_{\perp \alpha}}{\widetilde{\Omega}_{\alpha}^{2}}, \quad D_{\mathrm{A}}=\frac{\left(k_{\mathrm{e}}^{2}+k_{\mathrm{i}}^{2}\right) D_{\mathrm{e}} D_{\mathrm{i}}}{k_{\mathrm{e}}^{2} D_{\mathrm{e}}+k_{\mathrm{i}}^{2} D_{\mathrm{i}}} .
$$

After substitution into (25) one has

$$
\left\langle\delta n_{\mathrm{e}}^{2}\right\rangle_{\mathrm{k} \omega}=2 n_{\mathrm{e}} \frac{k^{2} D_{\mathrm{A}}}{\left|\omega+\mathrm{i} k^{2} D_{\mathrm{A}}\right|^{2}} \frac{k_{\mathrm{i}}^{4} D_{\mathrm{i}}+k_{\mathrm{e}}^{4} D_{\mathrm{e}}}{\left(k_{\mathrm{e}}^{2}+k_{\mathrm{i}}^{2}\right)\left(k_{\mathrm{e}}^{2} D_{\mathrm{e}}+k_{\mathrm{i}}^{2} D_{\mathrm{i}}\right)} .
$$

In the equilibrium case $\left(T_{\mathrm{e}}=T_{\mathrm{i}}\right)$ equation (57) reduces to

$$
\left\langle\delta n_{\mathrm{e}}^{2}\right\rangle_{\mathbf{k} \omega}=n_{\mathrm{e}} \frac{k^{2} D_{\mathrm{A}}}{\left|\omega+\mathrm{i} k^{2} D_{\mathrm{A}}\right|^{2}} .
$$

Similarly, it is easy to show that

$$
\left\langle\delta n_{\mathrm{i}}^{2}\right\rangle_{\mathbf{k} \omega}=2 n_{\mathrm{i}} \frac{k^{2} D_{\mathrm{A}}}{\left|\omega+\mathrm{i} k^{2} D_{\mathrm{A}}\right|^{2}} \frac{k_{\mathrm{i}}^{4} D_{\mathrm{i}}+k_{\mathrm{e}}^{4} D_{\mathrm{e}}}{\left(k_{\mathrm{e}}^{2}+k_{\mathrm{i}}^{2}\right)\left(k_{\mathrm{e}}^{2} D_{\mathrm{e}}+k_{\mathrm{i}}^{2} D_{\mathrm{i}}\right)} .
$$

Thus, we see that particle interaction leads to renormalization of the diffusion coefficients and to equalizing of the diffusive fluxes for particles of different species.

In the case of a strong magnetic field $\left(\Omega_{\alpha} \gg \beta_{\perp \alpha}\right)$ the ionic diffusion coefficient is much larger than the electronic one. It follows from the above relation that

$$
D_{\mathrm{A}} \sim D_{\mathrm{e}} \ll D_{\mathrm{i}}
$$

i.e. combined interaction suppresses the ionic diffusion.

\section{Acknowledgement}

This work was partially supported by grant No. 252/46 of the state fund of the fundamental researches of Ukraine. 


\title{
References
}

1. Gresillon D. et al. // Plasma Physics and Controlled Fusion, 1992, vol. 34, p. 1985-1992.

2. Villian J.P. et al. // J. Atmospherie and Terrestrial Phys., 1996, vol. 58, p. 943-958.

3. Gresillon D., Tomchuk B.P. // ECA, 1999, vol. 23J, p. 1045-1048.

4. Chandrasekhar S. // Rev. Mod. Phys., 1943, vol. 15, p. 1.

5. Schram P.P.J.M., Sitenko A.G., Zagorodny A.G. // ECA, 1999, vol. 23J, p. 888-891.

6. Zagorodny A., Weiland J. // Physics of Plasmas, 1999, vol. 6, p. 23-59.

7. Sitenko A.G., Zagorodny A.G. // Ukr. J. Phys., 1995, vol. 40, p. 390.

\section{Великомасштабні флуктуації та дифузія частинок поперек зовнішнього магнітного поля в турбулентній плазмі}

\author{
А.Загородній, І.Голод \\ Інститут теоретичної фізики ім. М.М.Боголюбова НАН України, \\ 03143 Київ, вул.Метрологічна, 14-б \\ Отримано 17 квітня 2000 р.
}

Розроблено кінетичну теорію електромагнітних флуктуацій у турбулентній плазмі за наявності зовнішнього магнітного поля з урахуванням впливу випадкових гідродинамічних рухів на динаміку флуктуацій. Обчислено функції діелектричного відгуку і кореляційні функції ланжевенових джерел та отримано загальні вирази для спектрів флуктуацій. Детально вивчено флуктуації пов'язані з дифузійним рухом частинок поперек зовнішнього магнітного поля.

Ключові слова: турбулентна дифузія, рівняння Фоккера-Планка, флуктуації

PACS: $05.40 .+j, 52.35 . R a, 25.65 . F f$ 